\title{
A New Power Law Linking the Speed to the Geometry of Tool-Tip Orientation in Teleoperation of a Robot-Assisted Surgical System
}

\author{
Or Zruya, Yarden Sharon, Student Member, IEEE, Hanna Kossowsky, Student Member, IEEE, \\ Fulvio Forni, Alex Geftler, and Ilana Nisky, Senior Member, IEEE
}

\begin{abstract}
Fine manipulation is important in dexterous tasks executed via teleoperation, including in robot-assisted surgery. Discovering fundamental laws of human movement can benefit the design and control of teleoperated systems, and the training of their users. These laws are formulated as motor invariants, such as the well-studied speed-curvature power law. However, while the majority of these laws characterise translational movements, fine manipulation requires controlling the orientation of objects. This subject has received little attention in human motor control studies. Here, we report a new power law linking the speed to the geometry in orientation control - humans rotate their hands with an angular speed that is exponentially related to the local change in rotation direction. We demonstrate this law in a teleoperated task performed by surgeons with a surgical robotics research platform. Additionally, we show that the law's parameters change slowly with the surgeons' training, and are robust within participants across task segments and repetitions. The fact that this power law is a robust motor invariant suggests that it may be an outcome of sensorimotor control. It also opens questions about the nature of this control and how it can be harnessed for better control of human-teleoperated robotic systems.
\end{abstract}

\section{INTRODUCTION}

In many teleoperated robot-assisted applications, users perform tasks requiring fine manipulations in six degrees of freedom - the translation and the rotation of objects (Fig. 1). For example, in Robotic-Assisted Minimally Invasive Surgeries (RAMIS), surgeons teleoperate robotic manipulators to control instruments inside a patient's body with which they manipulate objects such as tissue and needles. Understanding how humans coordinate the movement of their hands can be beneficial in the design and control of teleoperated systems, and the training of their users. It can also be informative for the design of path planners and controllers for systems that operate together with human users by making these systems more human-like and thus predictable [1], [2], [3].

*This work was supported by the ISF (grant number 327/20), by the Helmsley Charitable Trust through the Agricultural, Biological and Cognitive Robotics Initiative, the Marcus Endowment Fund both at Ben-Gurion University of the Negev, and the Israeli MOST within the Israel-Italy Virtual lab on artificial somatosensation.

Or Zruya, Yarden Sharon, Hanna Kossowsky and Ilana Nisky are with the Department of Biomedical Engineering and the Zlotowsky Center for Neuroscience, Ben-Gurion University of the Negev, Beer Sheva, Israel. orzrepost.bgu.ac.il, shayarepost.bgu.ac.il, hannako@post.bgu.ac.il, nisky@bgu.ac.il

Alex Geftler is with the Department of Orthopedic Surgery, Soroka Medical Center, Ben-Gurion University of the Negev, Beer Sheva, Israel. ageftleregmail.com

Fulvio Forni and Ilana Nisky are with the Department of Engineering, University of Cambridge, Cambridge CB2 1PZ, U.K. f. fornideng.cam.ac.uk
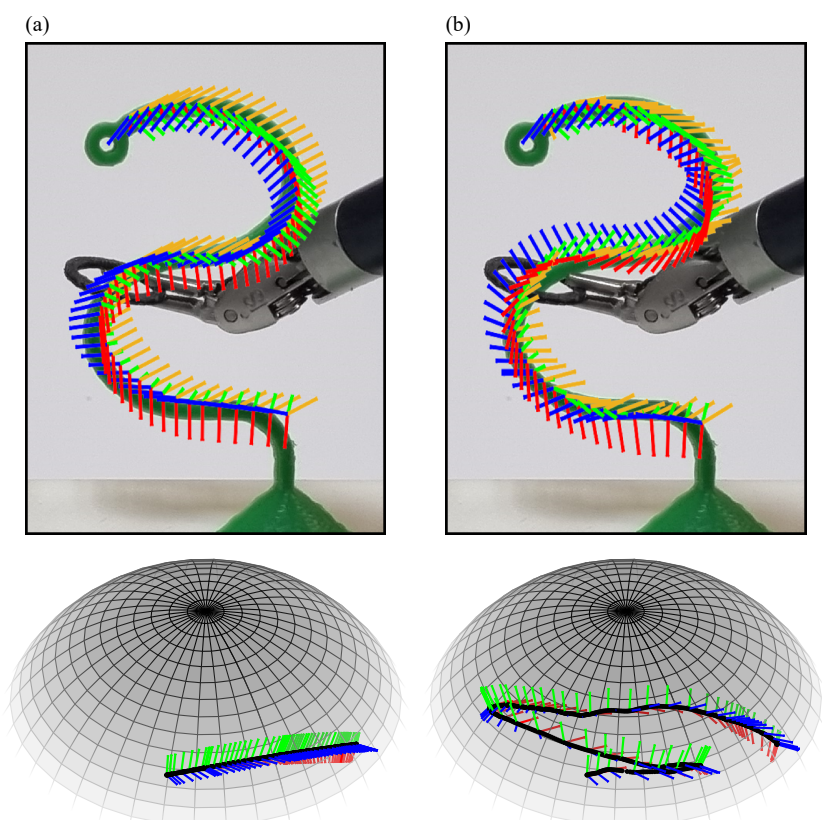

- orientation sample

instantaneous rotation axis

Fig. 1. Analysis of angular speed and local geometry of the orientation path of a teleoperated robot-assisted surgical instrument's tip. The top row presents the transfer of a ring on a wire, and the red, green and blue arrows represent a moving frame attached to the rotating gripper of the tool-tip. The orange arrow represents the instantaneous rotation axis (represented in a fixed reference frame) for the transition between two subsequent frames. The spheres in the bottom row illustrate the same movements as samples of orientations (black dots) using quaternions in $\mathbb{H}_{1}$ (for intuition, we illustrate $\mathbb{H}_{1}$ on which unit quaternions lie using the 2 -sphere). The angular speed is determined by the spherical distance between consecutive orientations in $\mathbb{H}_{1}$, and the local curvature is determined by the curvature of the path's projection in $\mathbb{H}_{1}$ onto the tangential plane (see Methods). (a) The tool-tip rotates around a fixed instantaneous rotation axis, and therefore, it creates a geodesic in $\mathbb{H}_{1}$ (arc). (b) The instantaneous rotation axis varies, therefore, the orientation path is a non-geodesic.

To discover the building blocks of human movement control, experimental and computational approaches are used. For example, the hand is kinematically redundant, and the basis on which the nervous system chooses one trajectory (path and timing) from an infinite number of possibilities was thoroughly studied [4], [5], [6]. Motor invariants, sometimes also referred to as motor primitives, are robust patterns that can be quantified in human movements. They are typically observed across repetitions of the same movements within and between participants, and are considered to be a result of active control by the nervous system [4]. 
One such invariant is the straight path and bell-shaped velocity trajectory that characterise fast point-to-point movement, which may result from the optimization of movement smoothness [4]. Another prominent invariant, which was also linked to movement smoothness [7], is the speed-curvature power law [8] which describes a relation between the instantaneous linear speed $(v)$ of a two-dimensional translational movement and its local path curvature $(\kappa)$ :

$$
v=\alpha \kappa^{\beta} .
$$

The linear speed characterizes the movement in space and time, whereas the curvature is a pure geometrical property - the local deviation of the path from a straight line. The speed gain $(\alpha)$ is generally piece-wise constant [8], [9], and the exponent $(\beta)$ is negative and constant for similar paths.

This law was first reported for drawing of ellipses with an exponent value of $-1 / 3$ [8], and was later generalized to a spectrum of power laws whose exponent depends on the number of curvature oscillations per angle unit [10]. It was demonstrated in a variety of movements, including movements of the hand [8], feet [11], and even the head during walking [12]. The law is thought to rely on physiological mechanisms, but its origin remains controversial. Some studies argue that trajectory is planned within the central nervous system [7], [13], [14], [15]. Others attribute the law to lower levels of the sensorimotor system [16], or to biomechanics of the limbs [17]. The neural representation of the power law was also argued to affect perception - humans perceived animated motion as more natural when it obeyed the speed-curvature power law [18]. A similar representation may underlie the recent results suggesting that human-robot physical interaction is most efficient when the robot moves in accordance with the speed-curvature power law [19].

When movements in three dimensions were considered, the power law was extended to the speed-curvature-torsion power law [20] that accounts for deviation from a straight and a planar path. This extended power law was demonstrated in surgical suturing, and shown to be dependent on the level of user expertise and whether the task was performed via RAMIS or via open surgery techniques [21]. However, this is still not sufficient to account for fine manipulation in many real-life robotic applications, such as RAMIS [22], that involve both translation and rotation that may be independently controlled [23]. Indeed, while motor invariants are extensively studied in the control of translation, a similar understanding of the control of orientation is lacking.

Some studies have already started to fill the gap. For example, a study of planar path following revealed that users exploited more portions of arm redundancy when the task required the control of both position and orientation of the tool compared to position alone [24]. Similarly, in the context of RAMIS training, peg transfer with a change of orientation [25], ring-on-wire transfer [26], and needledriving [27], [28], were investigated. However, a fundamental computational understanding of human control of orientation, and subsequently, manipulation in six degrees of freedom, has yet to be developed.
In this study, we aimed to address this gap by quantitatively characterizing the relation between the spatio-temporal and spatial changes of orientation trajectories represented by unit quaternions in $\mathbb{H}_{1}$ (Fig. 1). We propose a new power law linking between the speed and the geometry in human control of movement orientation. We demonstrate this law by analyzing movements of surgical residents performing a ring tower transfer using the da Vinci Research Kit (dVRK, Fig. 2a-b), a RAMIS research platform [29]. This task is commonly used in training of robotic surgeons [30], and requires the manipulation of the position and the orientation of a ring held by the gripper of the teleoperated instrument.

The remainder of this paper is organized as follows. In Section II we present the mathematical representation of orientation, the definition of the power law, and the description of the data and the analysis. In Section III, we present the result of fitting the power law to the data, and validation studies. In Section IV, we discuss the results in relation to the translational power law and their implications in the context of RAMIS, the limitations of this study, and future studies. In Section V, we present our conclusions.

\section{METHODS}

\section{A. Mathematical formulation}

In this subsection we present the formulation of a new power law which links between the speed and the geometry of the orientation of teleoperated robotic instruments. The manipulation of a rigid body in three-dimensional space involves movement in six degrees of freedom. Three degrees of freedom are associated with translation - the movement of the origin of a reference frame that is attached to the body, and the other three are associated with rotation of the reference frame about some axis. We considered rotations represented by unit quaternions, denoted by $\mathbf{q} \in \mathbb{H}_{1}$. $\mathbb{H}_{1}$ is the space of unit quaternions and is also known as the 3sphere - a four-dimensional unit size sphere. This will be helpful when visualizing quaternions (Fig. 1, bottom row).

Quaternions are hyper-complex numbers comprised of a real part $s \in \mathbb{R}$ and an imaginary part $\mathbf{v}=[x, y, z] \in \mathbb{R}^{3}$, such that $\mathbf{q}=s+\mathbf{i} x+\mathbf{j} y+\mathbf{k} z, \mathbf{i}^{2}=\mathbf{j}^{2}=\mathbf{k}^{2}=\mathbf{i j k}=-1, \mathbf{i j}=\mathbf{k}, \mathbf{j i}=$ $-\mathbf{k}$. According to Euler's theorem, for any $\mathbf{q} \in \mathbb{H}_{1}$, there exists an angle $\theta \in(-\pi, \pi]$ and an axis $\hat{\mathbf{n}} \in \mathbb{R}^{3}$, such that:

$$
\mathbf{q}=\cos \theta / 2+\hat{\mathbf{n}} \sin \theta / 2,
$$

where $\mathbf{q}$ is the quaternion that takes a point in $\mathbb{R}^{3}$ and rotates it $\theta$ around $\hat{\mathbf{n}}$.

Let $\{\mathbf{q}\}_{i=1}^{N}$ be a discrete time $N$ samples orientation trajectory (e.g. of the tool-tip of the Patient Side Manipulators (PSMs) of a teleoperated surgical robot - see next section for details), such that $\mathbf{q}_{i}=\cos \theta_{i} / 2+\hat{\mathbf{n}}_{i} \sin \theta_{i} / 2$ is the quaternion that rotates all the vectors represented in a fixed frame $\left(\mathbf{q}_{I}=1\right)$ by an angle $\theta_{i}$ around an axis $\hat{\mathbf{n}}_{i}$. We denote the discrete time trajectory of the transition quaternion as $\left\{\mathbf{q}^{\delta}\right\}_{i=1}^{N-1}$, such that $\mathbf{q}_{i}^{\delta}=\cos \theta_{i}^{\delta} / 2+\hat{\mathbf{n}}_{i}^{\delta} \sin \theta_{i}^{\delta} / 2$ is the quaternion that rotates $\mathbf{q}_{i}$ to $\mathbf{q}_{i+1}$ with an instantaneous angle $\theta_{i}^{\delta}$ around an instantaneous axis $\hat{\mathbf{n}}_{i}^{\delta}$ :

$$
\mathbf{q}_{i+1}=\mathbf{q}_{i}^{\delta} \mathbf{q}_{i},
$$


where $\delta$ is used to indicate incremental, small transition between $\mathbf{q}_{i}$ and $\mathbf{q}_{i+1}$. The angular speed at sample $i\left(\omega_{i}\right)$ quantifies the spatio-temporal change of the PSM tool-tip's orientation when it rotates about its center of mass:

$$
\omega_{i}=\theta_{i}^{\delta} / \delta t_{i}
$$

where $\delta t_{i}=t_{i+1}-t_{i}$ is the time sampling interval. The curvature at sample $i\left(\lambda_{i}\right)$ quantifies the spatial change of the instantaneous axis of rotation as the local curvature of a curve $\{\mathbf{q}\}_{i=1}^{N} \in \mathbb{H}_{1}[31]$ :

$$
\lambda_{i}=\left\|\mathbf{q}_{i}^{\prime \prime}-\left(\mathbf{q}_{i}^{\prime \prime} \cdot \mathbf{q}_{i}\right) \mathbf{q}_{i}\right\|,
$$

where $\|\cdot\|$ is the 2-norm. We approximate the second derivative of a quaternion curve at sample $i$ as in [31], [32]:

$$
\mathbf{q}_{i}^{\prime \prime}=\frac{\mathbf{q}_{i-1}-2 \mathbf{q}_{i}+\mathbf{q}_{i+1}}{l^{2}\left(\mathbf{q}_{i}\right)},
$$

where $l\left(\mathbf{q}_{i}\right)=\cos ^{-1}\left(\mathbf{q}_{i-1} \cdot \mathbf{q}_{i}\right) / 2+\cos ^{-1}\left(\mathbf{q}_{i} \cdot \mathbf{q}_{i+1}\right) / 2$. Eq. 5 originates from the projection of a curve in $\mathbb{H}_{1}$ onto the tangential plane, so it promises that a geodesic in $\mathbb{H}_{1}$ is characterized with $\lambda=0$ along the great arc.

The proposed power law relates the angular speed of the tool-tip with the local curvature of its path:

$$
\omega=\alpha \lambda^{\beta} \text {. }
$$

\section{B. Dataset}

To demonstrate the proposed power law in a natural orientation control task, we analyzed recordings of a teleoperated bi-manual ring tower transfer task (Fig. 2c) performed with the dVRK (Fig. 2a-b). Specifically, we analyzed the kinematics of the patient side manipulators (PSMs) that were used to perform this task. This data is part of a large dataset that was collected at the Biomedical Robotics Lab at Ben-Gurion University of the Negev as part of a long-term surgical skill learning study.

18 surgical residents from the Soroka Medical Center participated in the experiment after signing an informed consent form approved by the Human Participants Research Committee of Ben-Gurion University of the Negev, Beer Sheva, Israel. The participants were seated in front of a surgeon's console of the dVRK [33] and used the Master Tool Manipulators (MTMs) to control two PSMs (Fig. 2a). Each participant took part in four sessions of the experiment; each session was comprised of three trials that occurred before, during, and after a hospital shift. The gap between two consecutive sessions was approximately one month.

Each trial consisted of four ring tower transfers (Fig. 2c) - two vertical towers $\left(\mathrm{R}_{\mathrm{v}}, \mathrm{L}_{\mathrm{v}}\right)$ and two horizontal towers $\left(\mathrm{R}_{\mathrm{h}}, \mathrm{L}_{\mathrm{h}}\right)$ relative to the task board (see Video 1 for a demonstration of the task). At the beginning of each trial, the participants grasped a ring at the bottom of tower $R_{v}$ using the right PSM, and were instructed to trace the wire without touching it and extract the ring at the top of the tower. Following that, participants transferred the ring to the left PSM, inserted it through tower $\mathrm{L}_{\mathrm{h}}$ without touching the wire and placed it after the bulge. Next, participants used the
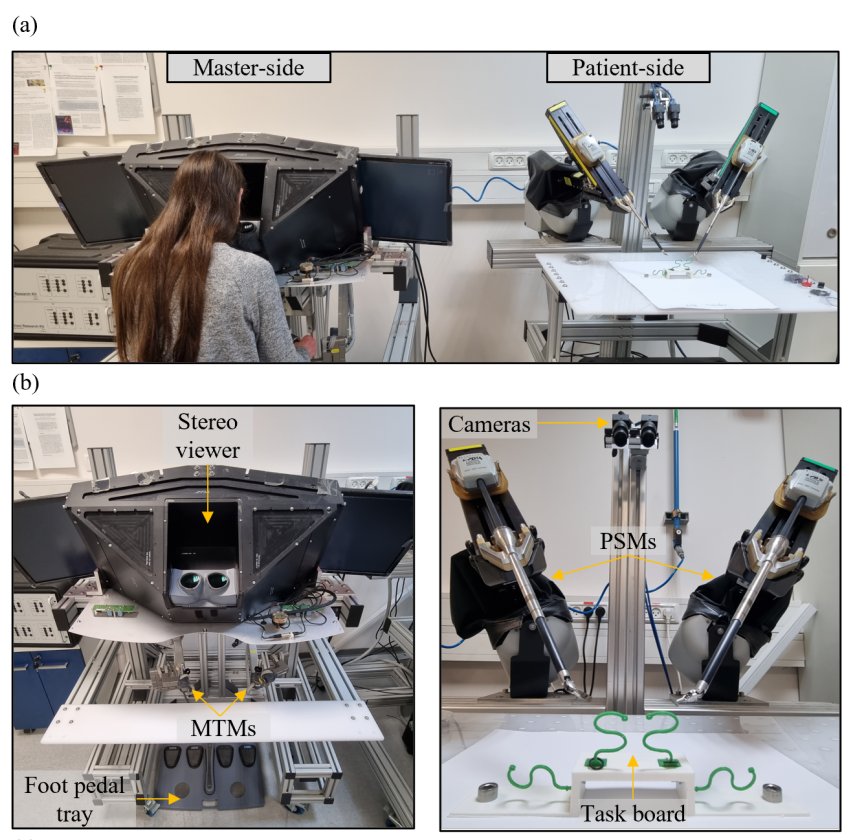

(c)

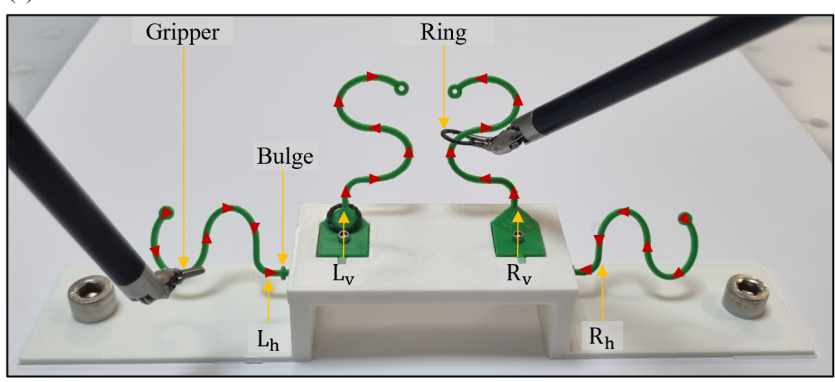

Fig. 2. Experimental setup and task. (a) The dVRK is a teleoperation system with master and patient sides. (b) The master side consists of stereo 3D viewer, MTMs and foot pedal tray. The patient side consists of cameras, PSMs and task board. (c) Participants performed four transfers of the ring in each trial by grasping it using the gripper of the PSMs. The right PSM was used to extract a ring from tower $R_{v}$ and insert it through tower $R_{h}$, and the left PSM was used to extract a ring from tower $L_{v}$ and insert it through tower $L_{h}$. The red arrows depict the instructed ring paths.

left PSM to grasp and extract a second ring from tower $\mathrm{L}_{\mathrm{v}}$ in a similar manner, transfer it to the right PSM and insert it through tower $R_{h}$. All the participants watched an instructional video that explained the task and showed one successful performance of the task. Successful performance required no contact of the ring with all the wires, but the trials were not interrupted even if contact was made. Participants were also instructed to avoid ring drops and PSMs collisions with the task board and between themselves, and were measured on the amount of time it took them to complete the task.

\section{Data analysis}

The orientation trajectories of the tool-tips of both PSMs were recorded as quaternions at $100[\mathrm{~Hz}]$. We only removed tower segments from the analysis in which participants failed to complete the task due to system malfunction and in which the participants dropped the ring or used the opposite side 
PSM than the one instructed. A total of 21 tower segments were removed (2.43\% of all tower segments).

The data analysis was done using a custom written MATLAB code (The MathWorks, Natick, Massachusetts, USA). We also recorded the video stream of all the trials at 35 $[\mathrm{Hz}]$. We low-pass filtered the orientation using spherical linear interpolation [31]. Each trial was manually segmented into the four ring tower transfers using the video of the trial. The transfers at towers $R_{v}$ and $L_{v}$ began when the PSM's jaw grasped the ring and ended when the ring was extracted. The transfers at towers $R_{h}$ and $L_{h}$ began when the ring was inserted into the tower and ended when it was placed after the bulge.

For each sample we calculated the angular speed $\left(\omega_{i}\right)$ and the local curvature $\left(\lambda_{i}\right)$. Next, for all the samples in each tower segment, we fit a log-log linear regression model:

$$
\log \omega_{i}=\log \alpha+\beta \log \lambda_{i}
$$

where $\log$ is the natural logarithm. To assess the goodness of fit of the proposed power law, for each tower segment, we calculated the variance explained by the linear model of the log-log transformed data $\left(\mathrm{R}^{2}\right)$, and evaluated its mean and standard deviation over all participants, sessions, trials and tower segments. To asses the variability of the model parameters and explained variance, we used the coefficient of variation (the standard deviation divided by the absolute mean). To show that the law's parameters are consistent throughout all task repetitions, we calculated the bootstrap 95\% confidence interval (CI) for the means of $\alpha$ and $\beta$ in each session, trial, and tower.

\section{Validation studies}

We made several efforts to assure that the power law is not a consequence of the way we estimate the angular speed and curvature from the data. First, to verify that the model parameters are not sensitive to the sampling rate of the orientation trajectory, we downsampled the data to 50 $[\mathrm{Hz}]$ and $33[\mathrm{~Hz}]$, and compared the distributions of the fit parameters and the explained variance.

Next, we manipulated a recorded orientation trajectory by sampling the quaternions at close to equal arc-lengths in $\mathbb{H}_{1}$. By assigning the original timestamps to the manipulated data, we generated a trajectory with a similar path, yet an angular speed that violates the power law. In addition, we simulated an orientation trajectory (not based on the dataset) that is inconsistent with the power law - it has two curvature oscillations and a nearly constant angular speed. We then fit the power law parameters to these manipulated and simulated examples of data, and verified that in these cases that clearly violate the power law, we indeed do not find a good fit.

\section{RESULTS}

\section{A. Power law analysis}

Fig. 3 presents an example of the analysis of one ring tower transfer. In analysis of speed-curvature power laws, it is important not to fit the power law to geodesics (i.e. straight paths in the case of translation or fixed instantaneous rotation axes in the case of orientation) [34]. A geodesic in $\mathbb{H}_{1}$ (Fig. 1a) results in an arc in the path of the quaternion axis $\hat{\mathbf{n}}$ (Eq. 2). The example path in Fig. 3a (top sphere) clearly deviates from an arc, implying the existence of a spatial change of orientation. Accordingly, the dispersed instantaneous rotation axis path in the bottom sphere indicates a change in the direction of rotation. Large spherical distance between consecutive instantaneous rotation axes implies a large curvature, and vice versa. The log-transformed curvature values in the data range from $\log \lambda=-2.06$ to $\log \lambda=15.42$, with an overall first order statistics of $\log \lambda=$ $4.73 \pm 1.86$ (mean \pm standard deviation). The large range of values indicates that we do not fit the model to geodesics (i.e. $\lambda>0$ ).

Examining the angular speed and curvature trajectories in Fig. $3 b$ clearly reveals that the angular speed is low when the local curvature is high, and vice versa. This is additionally supported by the log-log regression that is depicted in Fig. 3c, which presents a speed gain $\alpha=3.31[\mathrm{rad} / \mathrm{sec}]$, an exponent $\beta=-0.52$ and a good fit to the data with $\mathrm{R}^{2}=0.8$. We applied a similar analysis in each ring tower segment. The distributions of the fit parameters to the data sampled at $100[\mathrm{~Hz}]$ and the explained variance from all the segments (including different participants, sessions, trials, and tower segments) are depicted in Fig. 4. Both the speed gain and the exponent are narrowly distributed around the mean (the coefficient of variation was 0.23 for the speed gain and 0.09 for the exponent). In addition, the model resulted in a consistently high explained variance, which was also narrowly distributed (the coefficient of variation was 0.06 ). The explained variance was similar to the values that were previously reported in studies of the speed-curvature power law in translational movements [20], [35]. This analysis suggests that, consistently with the proposed power law in Eq. 7, the angular speed was exponentially related to the local curvature with a speed gain of $\alpha=3.39 \pm 0.78[\mathrm{rad} / \mathrm{sec}]$, and an exponent of $\beta=-0.54 \pm 0.05$. The variance explained by the model was $\mathrm{R}^{2}=0.81 \pm 0.05$.

In addition to proposing and demonstrating the adherence of rotational movements to the new power law, we wished to assess whether the parameters of the power law are consistent across executions of the task in different sessions, trials and tower segments. Since this is the first study to report the power law, we did not support this analysis by a statistical model, but rather present the distributions, their means and bootstrap 95\% CIs. Fig. 5 presents the fit parameters in each session (1-4), trial (1-3) and tower $\left(\mathrm{R}_{\mathrm{v}}, \mathrm{R}_{\mathrm{h}}, \mathrm{L}_{\mathrm{v}}, \mathrm{L}_{\mathrm{h}}\right)$, in the left, middle, and right panels, respectively. We averaged the parameters separately over each session, trial and tower, and estimated the bootstrap 95\% CI for the means (asterisks and error bars). The union of all CIs (gray shaded area) is $[3.03,3.66][\mathrm{rad} / \mathrm{sec}]$ for the speed gain and $[-0.57,-0.52]$ for the exponent. The similarities between CIs suggests that the model's parameters were quite robust with respect to these factors. There seems to be a trend of decrease in the speed gain and increase in the exponent with the progression of the sessions, but the change is very small (an average 


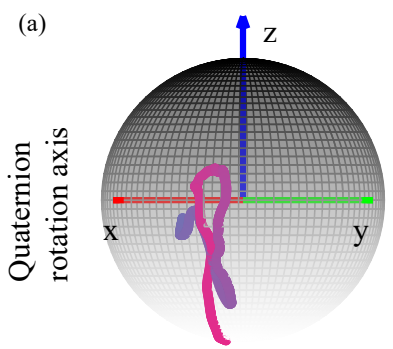

(b)
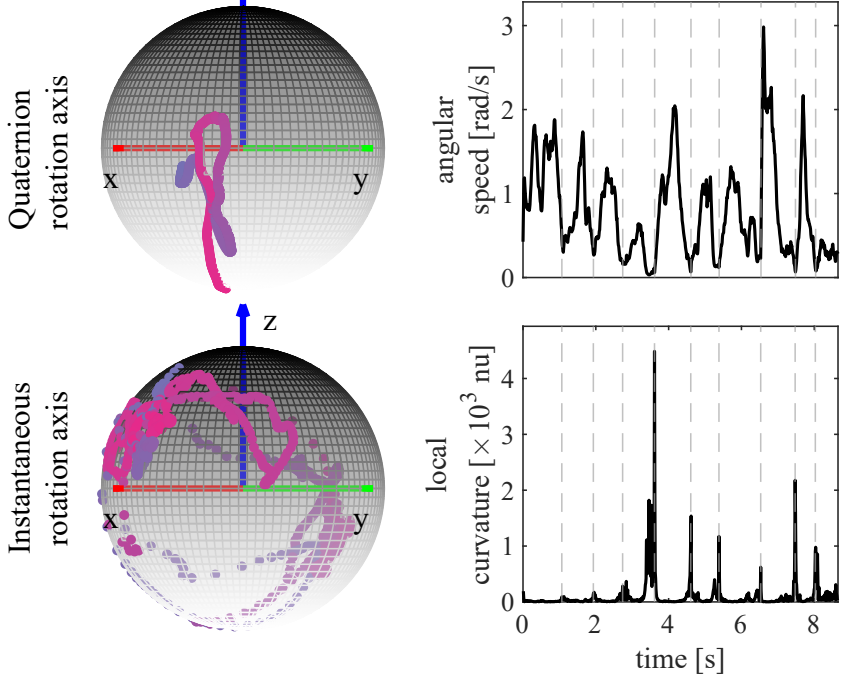

(c)

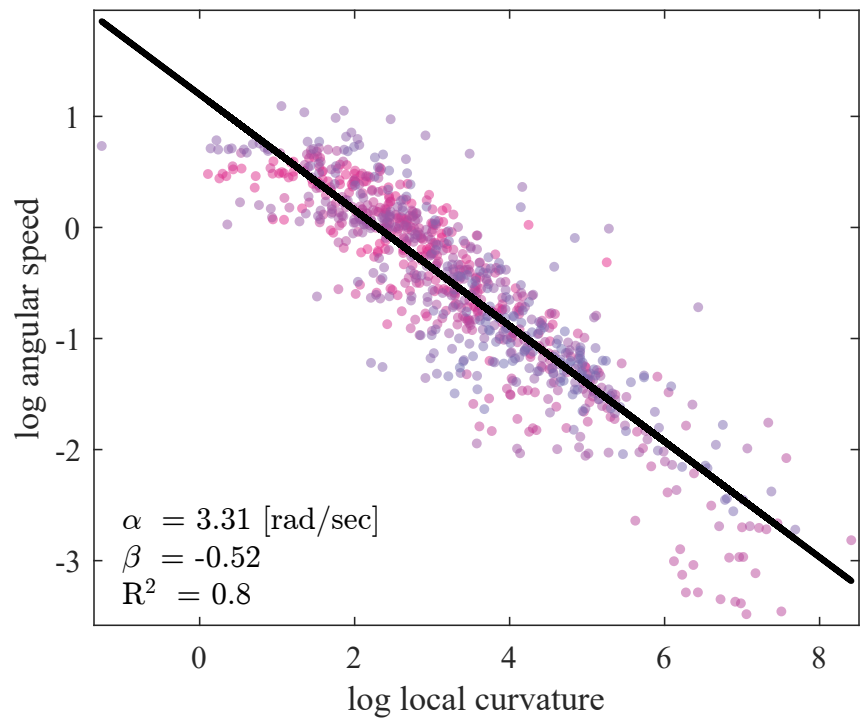

Fig. 3. An example of a ring tower transfer analysis. (a) An extrinsic reference frame is shown as red, green and blue arrows. The color of the points changes from pink to purple to indicate time progression. The tool-tip did not follow a geodesic in $\mathbb{H}_{1}$. This is observed in the quaternion rotation axis path ( $\hat{\mathbf{n}}$, top sphere) that deviates from an arc, and in the instantaneous rotation axis path ( $\hat{\mathbf{n}}^{\delta}$, bottom sphere) that is widely dispersed. (b) Angular speed (top) and local curvature (bottom) of the tool-tip orientation trajectory. The dashed lines indicate peaks in the local curvature that correspond to valleys in the angular speed. nu - no units. (c) The log-log regression model (black line) is fit to the data (dots). The model's parameters and explained variance are in the inset.
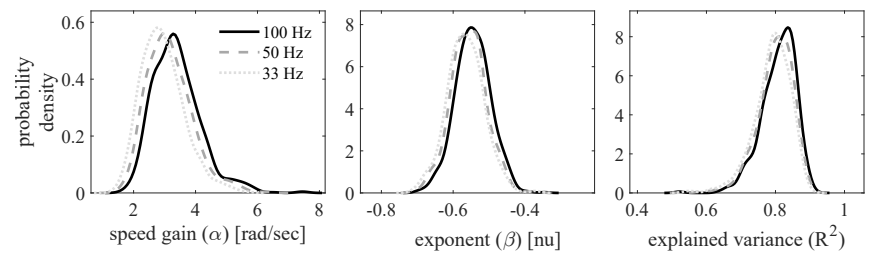

Fig. 4. The distributions of the fit model parameters, and the effect of sampling rate on these distributions. Downsampling the data slightly shifted the distribution of the speed gain with sampling rate, yet it nearly did not change the distributions of the exponent and the explained variance.

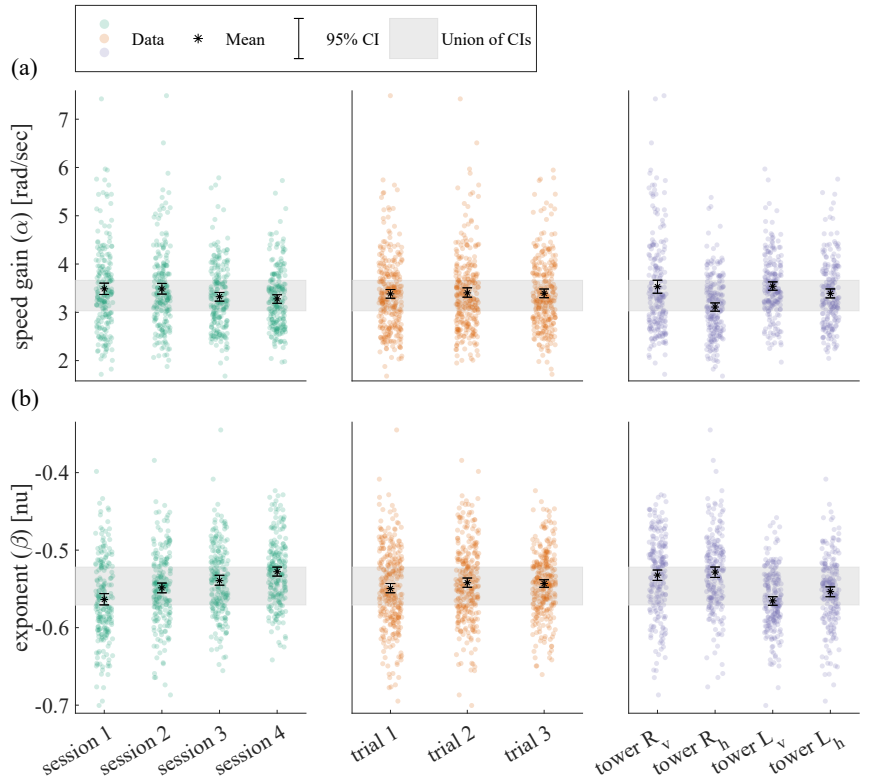

Fig. 5. Summary of the power law parameters. The asterisks show the mean of the speed gain (a) and exponent (b) in each session, trial and tower segment (left, middle and right panels). The error bars indicate the $95 \%$ bootstrap CI for the mean. The gray shaded area marks the union of all CIs for each model parameter.

decrease per session of $0.07[\mathrm{rad} / \mathrm{sec}]$ in the speed gain, and an average increase per session of 0.01 in the exponent).

\section{B. Validation studies}

One possible criticism to the validity of the proposed power law is that it could be a result of the sampling process [36]. If this were the case, we would expect the parameters of the power law to depend on the sampling rate. Hence, we downsampled the data to $50[\mathrm{~Hz}]$ and $33[\mathrm{~Hz}]$ and repeated the power law analysis. Fig. 4 shows the distributions of the fit parameters in these two cases compared to those of the original sampled data. We observed a slight shift in the distribution of the speed gain with sampling rate $(50[\mathrm{~Hz}]$ : $3.16 \pm 0.74,33[\mathrm{~Hz}]: 2.94 \pm 0.69)$, and a nearly identical distribution of the exponent $(50[\mathrm{~Hz}]:-0.55 \pm 0.05,33$ $[\mathrm{Hz}]:-0.56 \pm 0.05)$ and of the explained variance $(50[\mathrm{~Hz}]$ : $0.8 \pm 0.05,33[\mathrm{~Hz}]: 0.79 \pm 0.05)$.

Another possible criticism is that the results are purely a computational consequence of the math used to compute the angular speed and local curvature [37]. If this were the case, any dataset that we would generate would yield a good fit of the power law, regardless of whether the actual trajectory and path obey the power law. To counter this argument, we manipulated an existing orientation trajectory by breaking the dependency between the speed and the geometry. Fig. $6 \mathrm{a}$ shows the results of the manipulated orientation trajectory, and the poor fit of the power law. In addition, we simulated an equally spaced orientation trajectory in $\mathbb{H}_{1}$, thus creating a curved path traveled in a constant angular speed (Fig. 6b). The generated path is the equivalent of an ellipse in an Euclidean space, which has two curvature oscillations, and similarly does not yield a good fit to the power law. 


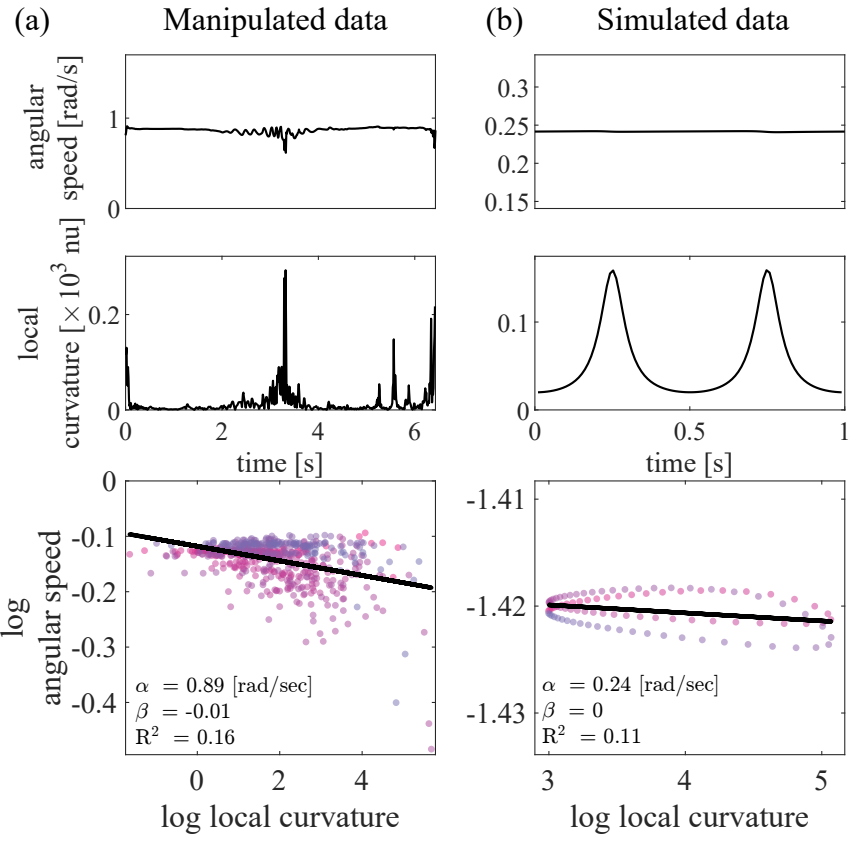

Fig. 6. Manipulated and simulated orientation trajectories that violate the power law. (a) We manipulated an orientation trajectory by sampling at close to equal arc-lengths resulting in a nearly constant angular speed (top) and curved path (middle) which violates the power law (bottom). (b) We simulated an orientation trajectory with nearly constant angular speed (top) and two curvature oscillations (middle) which is inconsistent with the power law (bottom). The fit model parameters and explained variance are in the inset. The color change is as in Fig. 3.

\section{DISCUSSION}

We presented a new power law which links the angular speed to the geometry of the tool-tip orientation in teleoperation of a robot-assisted surgical system. We analyzed kinematic data recorded during a teleoperated surgical training task - transfer of a ring along a wire. Unlike most motor control studies, we focused on the orientation of the controlled object, rather than on its position. We addressed the hypothesis of a power law linking between the local spatio-temporal change of orientation - the angular speed of the PSM's tool-tip when it rotates about its center of mass, and the local spatial change of orientation - the way a curve in $\mathbb{H}_{1}$ bends with respect to a geodesic. The model successfully accounted for $81 \%$ of the variance in the variables which spanned over at least two orders of magnitude, making the model a legitimate power law [38].

When formulating our new power law we were inspired by the decades long known speed-curvature law describing translational movements [8]. Our proposed law extends the original law to account for rotational movements. Indeed, the variance explained by our power law does not fall short from that found in translational 3D movements in the general [20], [35] as well as in the surgical context [21]. Similarly to the original power law, our results suggest the existence of an underlying control process that dictates dynamic commands in response to geometrical input, but it remains unknown in which level of the sensorimotor system this process is implemented, and which control methods are utilized. Even more so, it remains to discover in future work whether in general pose control tasks the translation and rotation are controlled separately (as was suggested for reaching movements [39]) or simultaneously [6], and whether linear and angular speed are affected in some more complex way by the local geometries of translational and rotational paths.

We found that the parameters of the power law - the speed gain and the exponent - are narrowly distributed across different participants and across different repetitions of the ring tower transfer task, indicating the robustness of the power law as a motor invariant of the control of orientation. Interestingly, the parameters of the translational speed-curvature power law were also previously proposed as a kinematic signature that can help in classifying surgical skill [21], [40]. Our primary goal in this study was to establish the power law, and hence, we did not wish to make any predictions about how its parameters may reflect skill. Nevertheless, in our posthoc analysis of the dependence of the parameters on the sessions, unlike [21], we observed only a slight decrease in the speed gain and a slight increase in the exponent over sessions, which could suggest that the law does not indicate surgical skill level. However, our data here spanned only four sessions of training over four months, and we cannot rule out the possibility that participants did learn. Indeed, the scale of learning is not established for rotational movements in relatively complex tasks. For example, parameters could change over years of learning and not months. Years of monitoring surgical skills or testing movements of surgeons at a wider range of expertise levels could reveal different changes of the fit parameters.

Similar to the speed-curvature power law in translations, the new law of movement that we propose suffers from singularity at points in $\mathbb{H}_{1}$ where $\lambda=0$, as the angular speed should become infinite. Though we did not force participants to rotate around a varying instantaneous axis, the analysis showed that they did (see example in Fig. 3). Nevertheless, low curvature points were observed. Future work is needed to construct an analytic framework that explains the new power law and predicts its extension to geodesics, similar to the minimum-jerk framework in the control of a rigid body's position [7]. This extension would allow a better understanding of rotation coordination across a wider range of local curvatures. In drawing, for example, points of inflection $\left(\kappa=0\left[\mathrm{~m}^{-1}\right]\right)$ are used to segment movements [9] into units of motor action and it remains to be determined if this segmented control hypothesis is evident in rotational data as well.

We used teleoperated RAMIS platform to study orientation control strategies of a remote controlled tool. The dynamics of any apparatus or environment constrain the human behavior, and even natural tool manipulation in thin air is constrained by the inertia and the friction of the tool, and to perform movements in viscous environments users have to account for the dynamics of the environment via adaptation [41]. Indeed, teleoperation has an effect on the measured movement [21], [42], potentially influencing the empirically derived parameters of the power law. However, 
so would any tool or manipulated object. Moreover, the structural significance of the power law lies in the robustness of the fit parameters across different repetitions of different participants, regardless of the experimental setup.

Of course, the good fit of our data to our proposed model does not indicate that it is necessarily true. Despite the fact that we did not constrain the tool to move according to any specific velocity profile, we considered the option that the results are either sampling-related or a trivial mathematical consequence [37]. Downsampling the orientation trajectory caused a small shift in the distribution of the speed gain, and nearly no change in the distribution of the exponent and explained variance. The relatively larger sensitivity of the speed gain to the sampling compared to the exponent and the explained variance may be explained by the relatively larger dispersion of the speed gain compared to the exponent and the explained variance also in the original fit. Therefore, we concluded that the power law model is insensitive to the sampling rate. Additionally, we manipulated the data to violate the power law and simulated data inconsistent with the power law that yielded a poor fit to the law, thus rejecting its triviality. Nevertheless, our lack of ability to refute the model does not mean it is true, as "all models are wrong, but some are useful" [43]. We nevertheless believe that our proposed power law has important implications in the context of RAMIS, as we outline in the next paragraphs.

Models of human motor control can be harnessed to a wide range of applications in human-centered control of robotic systems. Such systems may implement virtual fixtures software generated dynamic signals applied to the user [44]. In RAMIS, virtual fixtures can provide assistance to surgeons when navigating around delicate anatomical structures [45], [46]. A study of translational movements showed that such robotic assistance is most effective if it obeys laws of human movement, as users applied less force on the robot when the guided movement complied with the speed-curvature power law [19]. Accordingly, the new power law can be used to design guidance virtual fixtures in tasks with rotational movements.

Automation of surgical procedures in RAMIS is a promising field of research, due to its potential to provide better consistency across operations. For example, a recent study demonstrated an autonomous strategy for small bowel anastomosis in intestinal tissues [47]. While full surgical procedures are not yet performed autonomously, collaboration between surgeons and autonomous assistants is expected in the near future. Using biologically plausible movement patterns in robots make the robot's motion predictable and increase the efficiency of human-robot cooperation [1], [2], [3]. Therefore, we speculate that surgeons would be more comfortable operating in collaboration with robots that move in accordance with our proposed power law. Towards such biologically plausible movements, the new power law could be combined with the power law for translational movements observed in surgical data [21], [40], and yield trajectories that could account for all six degrees of freedom in space, and instruct rate according to the power laws.
However, it is important to note that clinical procedures involve more complex movements than what we studied here. They also involve time-dependant perturbations, such as the movement of the beating heart, and visual remapping due to tool or camera viewpoint misalignment [48]. These perturbations could highly affects the surgeon's performance [49], [50] and therefore surgical outcome. To be able to design virtual fixtures and autonomous surgical assistance with the help of motor invariants such as the power law that we report here, future studies are needed to test the effect of dynamic and visual perturbations on the parameters of the power law, and its applicability to more complex movements in contact with tissue.

\section{CONCLUSIONS}

We reported a novel power law describing the relation between the geometrical aspects of the orientation of a remote controlled tool and the angular speed profile used to follow that path. The power law analysis yielded a good fit of the experimental data to the model, with a consistent speed gain and exponent across participants and repetitions of the task. The results suggest the existence of a control process that translates the local spatial characteristics of the orientation of a rigid body into executable motor commands. We argue that knowledge of human control of orientation should be studied thoroughly and can be applied to the design of human-centered teleoperation systems in many applications requiring fine manipulation such as RAMIS.

\section{ACKNOWLEDGMENT}

We thank Tami Matus for administrative assistance and Noa Yamin, Sapir Goldring, Netali Auerbach, Nadav Amitai, Yuval Kassif and Ehud Zippin for collecting the data.

\section{REFERENCES}

[1] M. Huber, M. Rickert, A. Knoll, T. Brandt, and S. Glasauer, "Humanrobot interaction in handing-over tasks," in RO-MAN 2008 - The 17th IEEE International Symposium on Robot and Human Interactive Communication, Aug. 2008, pp. 107-112.

[2] A. Bisio, A. Sciutti, F. Nori, G. Metta, L. Fadiga, G. Sandini, and T. Pozzo, "Motor Contagion during Human-Human and Human-Robot Interaction," PLOS ONE, vol. 9, no. 8, p. e106172, Aug. 2014.

[3] E. De Momi, L. Kranendonk, M. Valenti, N. Enayati, and G. Ferrigno, "A Neural Network-Based Approach for Trajectory Planning in Robot-Human Handover Tasks," Frontiers in Robotics and AI, vol. 3, Jun. 2016.

[4] T. Flash and N. Hogan, "The coordination of arm movements: an experimentally confirmed mathematical model," Journal of Neuroscience, vol. 5, no. 7, pp. 1688-1703, Jul. 1985.

[5] E. Todorov, "Stochastic Optimal Control and Estimation Methods Adapted to the Noise Characteristics of the Sensorimotor System," Neural Computation, vol. 17, no. 5, pp. 1084-1108, May 2005.

[6] E. B. Torres and D. Zipser, "Reaching to grasp with a multi-jointed arm. i. computational model," Journal of Neurophysiology, vol. 88, no. 5, pp. 2355-2367, Nov. 2002.

[7] P. Viviani and T. Flash, "Minimum-jerk, two-thirds power law, and isochrony: converging approaches to movement planning," Journal of Experimental Psychology. Human Perception and Performance, vol. 21, no. 1, pp. 32-53, Feb. 1995.

[8] F. Lacquaniti, C. Terzuolo, and P. Viviani, "The law relating the kinematic and figural aspects of drawing movements," Acta Psychologica, vol. 54, no. 1, pp. 115-130, Oct. 1983.

[9] P. Viviani and M. Cenzato, "Segmentation and coupling in complex movements," Journal of Experimental Psychology. Human Perception and Performance, vol. 11, no. 6, pp. 828-845, Dec. 1985. 
[10] D. Huh and T. J. Sejnowski, "Spectrum of power laws for curved hand movements," Proceedings of the National Academy of Sciences, vol. 112, no. 29, pp. E3950-E3958, Jul. 2015.

[11] Y. P. Ivanenko, R. Grasso, V. Macellari, and F. Lacquaniti, "Twothirds power law in human locomotion: role of ground contact forces," Neuroreport, vol. 13, no. 9, pp. 1171-1174, Jul. 2002.

[12] S. Vieilledent, Y. Kerlirzin, S. Dalbera, and A. Berthoz, "Relationship between velocity and curvature of a human locomotor trajectory," Neuroscience Letters, vol. 305, no. 1, pp. 65-69, Jun. 2001.

[13] A. B. Schwartz and D. W. Moran, "Motor cortical activity during drawing movements: Population representation during lemniscate tracing," Journal of Neurophysiology, vol. 82, no. 5, pp. 2705-2718, 1999.

[14] M. Karklinsky and T. Flash, "Timing of continuous motor imagery: the two-thirds power law originates in trajectory planning," Journal of Neurophysiology, vol. 113, no. 7, pp. 2490-2499, Apr. 2015.

[15] Y. Meirovitch, H. Harris, E. Dayan, A. Arieli, and T. Flash, "Alpha and Beta Band Event-Related Desynchronization Reflects Kinematic Regularities," Journal of Neuroscience, vol. 35, no. 4, pp. 1627-1637, Jan. 2015.

[16] S. Schaal and D. Sternad, "Origins and violations of the $2 / 3$ power law in rhythmic three-dimensional arm movements," Experimental Brain Research, vol. 136, no. 1, pp. 60-72, Jan. 2001.

[17] P. L. Gribble and D. J. Ostry, "Origins of the power law relation between movement velocity and curvature: modeling the effects of muscle mechanics and limb dynamics," Journal of Neurophysiology, vol. 76, no. 5, pp. 2853-2860, Nov. 1996.

[18] E. Dayan, A. Casile, N. Levit-Binnun, M. A. Giese, T. Hendler, and T. Flash, "Neural representations of kinematic laws of motion: Evidence for action-perception coupling," Proceedings of the National Academy of Sciences, vol. 104, no. 51, pp. 20 582-20587, Dec. 2007.

[19] P. Maurice, M. E. Huber, N. Hogan, and D. Sternad, "VelocityCurvature Patterns Limit Human-Robot Physical Interaction," IEEE Robotics and Automation Letters, vol. 3, no. 1, pp. 249-256, Jan. 2018.

[20] U. Maoz, A. Berthoz, and T. Flash, "Complex Unconstrained ThreeDimensional Hand Movement and Constant Equi-Affine Speed," Journal of Neurophysiology, vol. 101, no. 2, pp. 1002-1015, Feb. 2009.

[21] Y. Sharon and I. Nisky, "Expertise, Teleoperation, and Task Constraints Affect the Speed-Curvature-Torsion Power Law in RAMIS," Journal of Medical Robotics Research, vol. 03, no. 03n04, p. 1841008, Sep. 2018.

[22] A. M. Jarc and I. Nisky, "Robot-assisted surgery: an emerging platform for human neuroscience research," Frontiers in Human Neuroscience, vol. 9, p. 315, Jun. 2015.

[23] J. F. Soechting and M. Flanders, "Parallel, interdependent channels for location and orientation in sensorimotor transformations for reaching and grasping," Journal of Neurophysiology, vol. 70, no. 3, pp. 11371150, Sep. 1993.

[24] J. Buzzi, E. De Momi, and I. Nisky, "An Uncontrolled Manifold Analysis of Arm Joint Variability in Virtual Planar Position and Orientation Telemanipulation," IEEE Transactions on Biomedical Engineering, vol. 66, no. 2, pp. 391-402, Feb. 2019.

[25] M. M. Coad, A. M. Okamura, S. Wren, Y. Mintz, T. S. Lendvay, A. M. Jarc, and I. Nisky, "Training in divergent and convergent force fields during 6-DOF teleoperation with a robot-assisted surgical system," in 2017 IEEE World Haptics Conference (WHC), Jun. 2017, pp. 195200.

[26] N. Enayati, A. M. Okamura, A. Mariani, E. Pellegrini, M. M. Coad, G. Ferrigno, and E. De Momi, "Robotic Assistance-as-Needed for Enhanced Visuomotor Learning in Surgical Robotics Training: An Experimental Study," in 2018 IEEE International Conference on Robotics and Automation (ICRA), May 2018, pp. 6631-6636.

[27] Y. Sharon, A. M. Jarc, T. S. Lendvay, and I. Nisky, "Rate of Orientation Change as a New Metric for Robot-Assisted and Open Surgical Skill Evaluation," IEEE Transactions on Medical Robotics and Bionics, vol. 3, no. 2, pp. 414-425, May 2021.

[28] I. Nisky, Y. Che, Z. F. Quek, M. Weber, M. H. Hsieh, and A. M. Okamura, "Teleoperated versus open needle driving: Kinematic analysis of experienced surgeons and novice users," in 2015 IEEE International Conference on Robotics and Automation (ICRA), May 2015, pp. 53715377.

[29] P. Kazanzides, Z. Chen, A. Deguet, G. S. Fischer, R. H. Taylor, and S. P. DiMaio, "An open-source research kit for the da Vinci® Surgical
System," in 2014 IEEE International Conference on Robotics and Automation (ICRA), May 2014, pp. 6434-6439.

[30] R. Smith, V. Patel, and R. Satava, "Fundamentals of robotic surgery: a course of basic robotic surgery skills based upon a 14-society consensus template of outcomes measures and curriculum development," The international journal of medical robotics and computer assisted surgery, vol. 10, no. 3, pp. 379-384, Sep. 2014.

[31] E. Dam, M. Koch, and M. Lillholm, "Quaternions, interpolation and animation,” Datalogisk Institut, Københavns Universitet, Tech. Rep., 1998.

[32] A. H. Barr, B. Currin, S. Gabriel, and J. F. Hughes, "Smooth interpolation of orientations with angular velocity constraints using quaternions," ACM SIGGRAPH Computer Graphics, vol. 26, no. 2, pp. 313-320, Jul. 1992.

[33] L. Bahar, Y. Sharon, and I. Nisky, "Surgeon-Centered Analysis of Robot-Assisted Needle Driving Under Different Force Feedback Conditions," Frontiers in Neurorobotics, vol. 13, Jan. 2020.

[34] E. Todorov and M. I. Jordan, "Smoothness Maximization Along a Predefined Path Accurately Predicts the Speed Profiles of Complex Arm Movements," Journal of Neurophysiology, vol. 80, no. 2, pp. 696-714, Aug. 1998.

[35] F. E. Pollick, U. Maoz, A. A. Handzel, P. J. Giblin, G. Sapiro, and T. Flash, "Three-dimensional arm movements at constant equi-affine speed," Cortex, vol. 45, no. 3, pp. 325-339, Mar. 2009.

[36] M. Zago, A. Matic, T. Flash, A. Gomez-Marin, and F. Lacquaniti, "The speed-curvature power law of movements: a reappraisal," Experimental Brain Research, vol. 236, no. 1, pp. 69-83, Jan. 2018.

[37] R. S. Marken and D. M. Shaffer, "The power law of movement: an example of a behavioral illusion," Experimental Brain Research, vol. 235, no. 6, pp. 1835-1843, Jun. 2017.

[38] M. P. H. Stumpf and M. A. Porter, "Critical Truths About Power Laws," Science, vol. 335, no. 6069, pp. 665-666, Feb. 2012.

[39] J. Fan, J. He, and S. I. H. Tillery, "Control of hand orientation and arm movement during reach and grasp," Experimental brain research, vol. 171, no. 3, pp. 283-296, May. 2006.

[40] A. French, T. S. Lendvay, R. M. Sweet, and T. M. Kowalewski, "Predicting surgical skill from the first $\mathrm{N}$ seconds of a task: value over task time using the isogony principle," International Journal of Computer Assisted Radiology and Surgery, vol. 12, no. 7, pp. 11611170, Jul. 2017.

[41] R. Shadmehr and F. A. Mussa-Ivaldi, "Adaptive representation of dynamics during learning of a motor task," Journal of Neuroscience, vol. 14, no. 5, pp. 3208-3224, May. 1994.

[42] I. Nisky, A. M. Okamura, and M. H. Hsieh, "Effects of robotic manipulators on movements of novices and surgeons," Surgical Endoscopy, vol. 28, no. 7, pp. 2145-2158, Jul. 2014.

[43] G. E. P. Box, "Science and Statistics," Journal of the American Statistical Association, vol. 71, no. 356, pp. 791-799, 1976.

[44] L. B. Rosenberg, "The Use of Virtual Fixtures as Perceptual Overlays to Enhance Operator Performance in Remote Environments." STANFORD UNIV CA CENTER FOR DESIGN RESEARCH, Tech. Rep., Sep. 1992.

[45] J. J. Abbott, P. Marayong, and A. M. Okamura, "Haptic Virtual Fixtures for Robot-Assisted Manipulation," in Robotics Research, ser. Springer Tracts in Advanced Robotics, S. Thrun, R. Brooks, and H. Durrant-Whyte, Eds. Berlin, Heidelberg: Springer, 2007, pp. 4964.

[46] M.-A. Vitrani, C. Poquet, and G. Morel, "Applying Virtual Fixtures to the Distal End of a Minimally Invasive Surgery Instrument," IEEE Transactions on Robotics, vol. 33, no. 1, pp. 114-123, Feb. 2017.

[47] H. Saeidi, J. D. Opfermann, M. Kam, S. Wei, S. Leonard, M. H. Hsieh, J. U. Kang, and A. Krieger, "Autonomous robotic laparoscopic surgery for intestinal anastomosis," Science Robotics, vol. 7, no. 62, p. eabj2908, Jan. 2022.

[48] L. H. Kim, C. Bargar, Y. Che, and A. M. Okamura, "Effects of masterslave tool misalignment in a teleoperated surgical robot," in 2015 IEEE International Conference on Robotics and Automation (ICRA), May 2015, pp. 5364-5370.

[49] Y. Sharon, D. Naftalovich, L. Bahar, Y. Refaely, and I. Nisky, "Combining Time-Dependent Force Perturbations in Robot-Assisted Surgery Training," arXiv:2105.03917 [cs], May 2021.

[50] C. Ames, A. J. Frisella, Y. Yan, P. Shulam, and J. Landman, "Evaluation of Laparoscopic Performance with Alteration in Angle of Vision," Journal of Endourology, vol. 20, no. 4, pp. 281-283, Apr. 2006. 